\title{
Automation in computer-aided cryptography: proofs, attacks and designs
}

\author{
Gilles Barthe ${ }^{1}$, Benjamin Grégoire ${ }^{3}$, César Kunz ${ }^{1,2}$, \\ Yassine Lakhnech ${ }^{4}$, and Santiago Zanella Béguelin ${ }^{5}$ \\ 1 IMDEA Software Institute \\ 2 Universidad Politécnica de Madrid \\ 3 INRIA Sophia Antipolis-Méditerranée \\ ${ }^{4}$ Université de Grenoble, France \\ ${ }^{5}$ Microsoft Research
}

CertiCrypt [3] and EasyCrypt [2] are machine-checked frameworks for proving the security of cryptographic constructions. Both frameworks adhere to the game-based approach $[9,6,8]$ to provable security [7], but revisit its realization from a formal verification pespective. More specifically, CertiCrypt and EasyCrypt use a probabilistic programming language pWHILE for expressing cryptographic constructions, security properties, and computational assumptions, and a probabilistic relational Hoare logic pRHL for justifying reasonings in cryptographic proofs. While both tools coincide in their foundations, they differ in their underlying technologies: CertiCrypt is implemented as a set of libraries in the Coq proof assistant, whereas EasyCrypt uses a verification condition generator for pRHL in combination with off-the-shelf SMT solvers and automated theorem provers. Over the last six years, we have used both tools to verify prominent examples of public-key encryption schemes, modes of operation, signature schemes, hash function designs, zero-knowledge proofs. Recently, we have also used both tools to certify the output of a zero-knowledge compiler [1].

The next challenge is to extend EasyCrypt with automated mechanisms for discovering proofs or attacks. As a first step in this direction, we have developed a front-end that searches for security proofs or attacks for public-key encryption schemes built from one-way trapdoor permutations and random oracles. Given a candidate scheme, the front-end first searches for attacks using a deducibility relation inspired from symbolic cryptography: if an attack is found, it outputs an attacker. If not, the front-end searches for game-based proofs that the scheme is secure: if a proof is found, it outputs a concrete security bound and an EasyCrypt script that can be verified independently. We have evaluated the applicability of the front-end on more than hundred variants of OAEP [5], a widely used padding scheme commonly used for strengthening RSA encryption: pleasingly, it proves most secure variants of OAEP and computes security bounds that match known bounds in many cases. In addition, we have used the front-end in combination with synthesis algorithms to explore the design space of the class of encryption schemes it covers. This has led to the discovery of ZAEP [4], a simplified variant of the OAEP padding scheme that can be used to strengthen RSA encryption with exponents 2 and 3 .

More information about the project can be found at: 
http://easycrypt.gforge.inria.fr

\section{References}

1. José Bacelar Almeida, Manuel Barbosa, Endre Bangerter, Gilles Barthe, Stephan Krenn, and Santiago Zanella Béguelin. Full proof cryptography: Verifiable compilation of efficient zero-knowledge protocols. In 19th ACM Conference on Computer and Communications Security, CCS 2012. ACM, 2012.

2. Gilles Barthe, Benjamin Grégoire, Sylvain Heraud, and Santiago Zanella Béguelin. Computer-aided security proofs for the working cryptographer. In Advances in Cryptology - CRYPTO 2011, volume 6841 of Lecture Notes in Computer Science, pages 71-90, Heidelberg, 2011. Springer.

3. Gilles Barthe, Benjamin Grégoire, and Santiago Zanella Béguelin. Formal certification of code-based cryptographic proofs. In 36th ACM SIGPLAN-SIGACT Symposium on Principles of Programming Languages, POPL 2009, pages 90-101, New York, 2009. ACM.

4. Gilles Barthe, David Pointcheval, and Santiago Zanella Béguelin. Verified security of redundancy-free encryption from Rabin and RSA. In 19th ACM Conference on Computer and Communications Security, CCS 2012. ACM, 2012. To appear.

5. Mihir Bellare and Phillip Rogaway. Optimal asymmetric encryption. In Advances in Cryptology - EUROCRYPT 1994, volume 950 of Lecture Notes in Computer Science, pages 92-111, Heidelberg, 1994. Springer.

6. Mihir Bellare and Phillip Rogaway. The security of triple encryption and a framework for code-based game-playing proofs. In Advances in Cryptology - EUROCRYPT 2006, volume 4004 of Lecture Notes in Computer Science, pages 409-426, Heidelberg, 2006. Springer.

7. Shafi Goldwasser and Silvio Micali. Probabilistic encryption. J. Comput. Syst. Sci., 28(2):270-299, 1984

8. S. Halevi. A plausible approach to computer-aided cryptographic proofs. Cryptology ePrint Archive, Report 2005/181, 2005.

9. Victor Shoup. Sequences of games: a tool for taming complexity in security proofs. Cryptology ePrint Archive, Report 2004/332, 2004. 